Relato de Caso / Case Report

\title{
Infiltração cutânea na leucemia megacariocítica aguda com expressão de CD56
} Cutaneous infiltration in acute megakaryocytic leukemia with CD56 expression

Mariela G. Farias ${ }^{1}$

Maristela B. Biermann ${ }^{2}$

Ana Lígia S. Matos ${ }^{3}$

Suzane Dal Bo ${ }^{1}$

Katia S. D. Santos ${ }^{1}$

\begin{abstract}
A LMA-M7 é um subtipo raro de leucemia mielóide aguda (LMA). Está freqüentemente associada a mielofibrose e representa um subtipo de mau prognóstico. Raramente apresenta infiltração em sítios extramedulares. O aspirado de medula óssea ou biópsia mostra uma população de células pleomórficas e basofílicas, que podem apresentar projeções citoplasmáticas. A utilização da imunofenotipagem é essencial para o diagnóstico de LMA-M7. O imunofenótipo característico apresenta uma população de células leucêmicas com ausência da maioria dos marcadores linfóides e mielóides de superfície, porém com expressão para os antígenos da linhagem megacariocítica: CD41a (complexo glicoprotéico IIb/IIIa), CD42b (glicoproteína Ib) e/ou CD61 (glicoproteína IIIa), ou antígeno relacionado ao fator VIII. Freqüentemente, a coloração citoquímica Sudan Black para os blastos megacariocíticos é negativa; neste caso, foi positiva para $40 \%$ das células analisadas. A presença de CD56, cuja expressão aberrante em algumas leucemias mielóides é indicativo de mau prognóstico, pode estar associada à infiltração da pele. Rev. bras. hematol. hemoter. 2007;29(2):185-188.
\end{abstract}

Palavras-chave: Leucemia megacariocítica aguda; citoquímica; imunofenotipagem; CD56; infiltração cutânea.

\section{Introdução}

A leucemia megacariocítica aguda (LMA-M7) é um subtipo raro de leucemia mielóide aguda (LMA), que foi recentemente incorporada na classificação Franco-AmericanaBritânica (FAB). ${ }^{1,2,3}$ Representa 3\% a 5\% dos casos de LMA e exibe uma curva de incidência de distribuição com um pico bimodal, ou seja, um pico em crianças menores de três anos e outro em idosos. ${ }^{1,3,5,6,7}$

A utilização apenas de critérios morfológicos e citoquímicos não é suficiente para um diagnóstico correto, pois são células morfologicamente indistinguíveis, com reações citoquímicas Sudan Black (SB) e mieloperoxidase negativas. ${ }^{1,2}$ Assim, a utilização de técnicas de imunofenotipagem é essencial para o diagnóstico diferencial, mostrando uma população de células leucêmicas com ausência da maioria dos marcadores linfóides e mielóides de superfície. Verifica-se que há expressão para os antígenos da linhagem megacariocítica: CD41a (complexo glicoprotéico IIb/IIIa), CD42b (glicoproteína Ib) e/ou CD61 (glicoproteína IIIa) ou antígeno relacionado ao fator VIII, permitindo uma classificação correta em $98 \%$ dos casos. ${ }^{2,4,6-10}$

O CD56 é uma molécula de adesão de célula neural (NCAM), encontrada nas células natural killer (NK) e subpopulações de células T. ${ }^{2,11}$ Na LMA está presente em $15 \%$ a $20 \%$ dos casos, sendo freqüentemente associado às leucemias de linhagem monocítica, a trissomia 8 , a t(8;21), $\mathrm{t}(15 ; 17)$ e ao rearranjo $11 \mathrm{q} 23$. $^{2}$ A expressão aberrante de CD56 pode predispor à infiltração leucêmica extramedular. ${ }^{12,13,14}$

Leucemia extramedular com infiltração na pele (leucemia cutis), é relativamente rara, mas é uma manifestação bem des-

${ }^{1}$ Unidade de Hematologia, Serviço de Patologia Clínica do Hospital de Clínicas de Porto Alegre (HCPA).

${ }^{2}$ Laboratório Central, Irmandade da Santa Casa de Misericórdia de Porto Alegre (ISCMPA).

${ }^{3}$ Instituto Nacional de Traumo-Ortopedia (INTO).

Correspondência: Mariela Granero Farias

Unidade de Hematologia do Serviço de Patologia Clínica

Hospital de Clínicas de Porto Alegre

Rua Ramiro Barcelos, 2350

90035-903 - Porto Alegre-RS - Brasil

Tel.: (51) 2101-8674

E-mail:mg.farias@yahoo.com.br 
crita na leucemia mielóide aguda, cujo diagnóstico é de importância clínica, pois determina um mau prognóstico. ${ }^{15,16,17}$ Infiltração cutânea raramente está associada a leucemia megacariocítica. ${ }^{15}$

Diante desses dados, este trabalho tem como objetivo relatar um caso de leucemia megacariocítica aguda com envolvimento extramedular (leucemia cutis), com marcador CD56 e coloração citoquímica Sudan Black (SB) positivos.

\section{Relato de caso}

Paciente do sexo masculino, 72 anos, chapeador aposentado, com história de alcoolismo, chegou ao consultório de Emergência do Hospital de Clínicas de Porto Alegre (HCPA), com queixa de disúria e febre. Pelo exame físico, revelou lesões nodulares subcutâneas disseminadas, sem prurido ou dor, sem perda ou ganho de peso significativo, negou alergias e tabagismo. Inicialmente suspeitou-se de leucemia linfóide aguda.

A história atual desse paciente foi neutropenia febril, hipertensão arterial sistêmica (HAS) e infecção do trato urinário (ITU).

Os achados hematológicos no sangue periférico foram: leucócitos 3,50 x103/ $\mu \mathrm{L}$ com $43 \%$ de blastos, hemoglobina 9,5 g/dL e plaquetas 105.000/ $\mu \mathrm{L}$; medula óssea hipercelular, infiltrada por $86 \%$ de blastos hipogranulares, pleomórficos, com projeções citoplasmáticas (Figura 1). Coloração Sudan Black positiva $40 \%$ (Figura 2). A imunofenotipagem demonstrou positividade para: CD45 (94\%), HLA-DR (93\%), CD56 (81\%) e CD61 (69\%) (Figura 3).

Identificou-se, também, uma pequena população de células, com fenótipo mielóide imaturo HLA-DR/CD13 (23\%), mieloperoxidase (11,5\%) e CD33 (5\%). O CD41 e o CD42 não foram examinados.

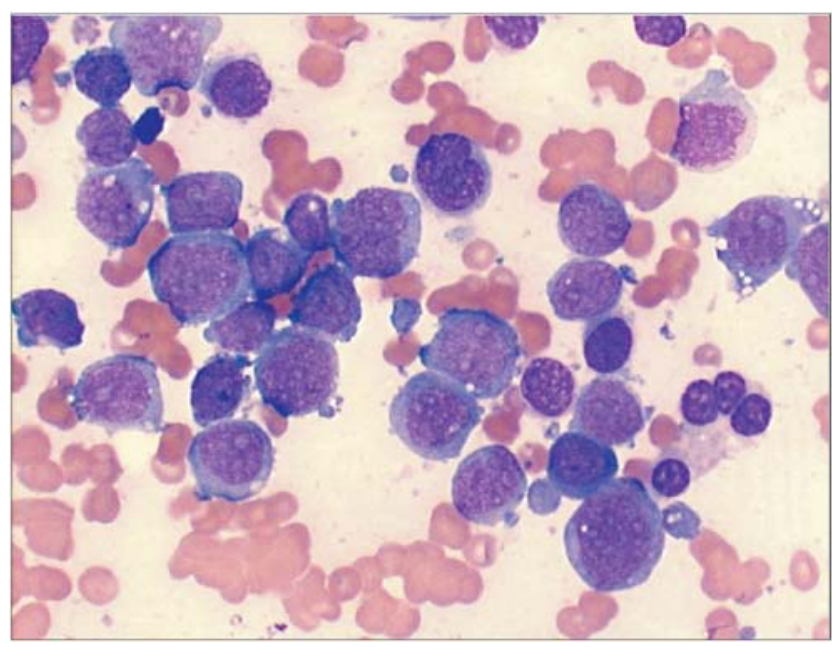

Figura 1. Distensão de medula óssea, corada com May-GrünwaldGiemsa (x500), exibe blastos com nucléolos evidentes, citoplasma basofílico, alguns com projeções citoplasmáticas e/ou vacúolos. Presença de macroplaquetas
A biópsia anatomopatológica (pele-tórax) revelou infiltrado mononuclear atípico consistente, com infiltração por leucemia (leucemia cutis) (Figura 4).

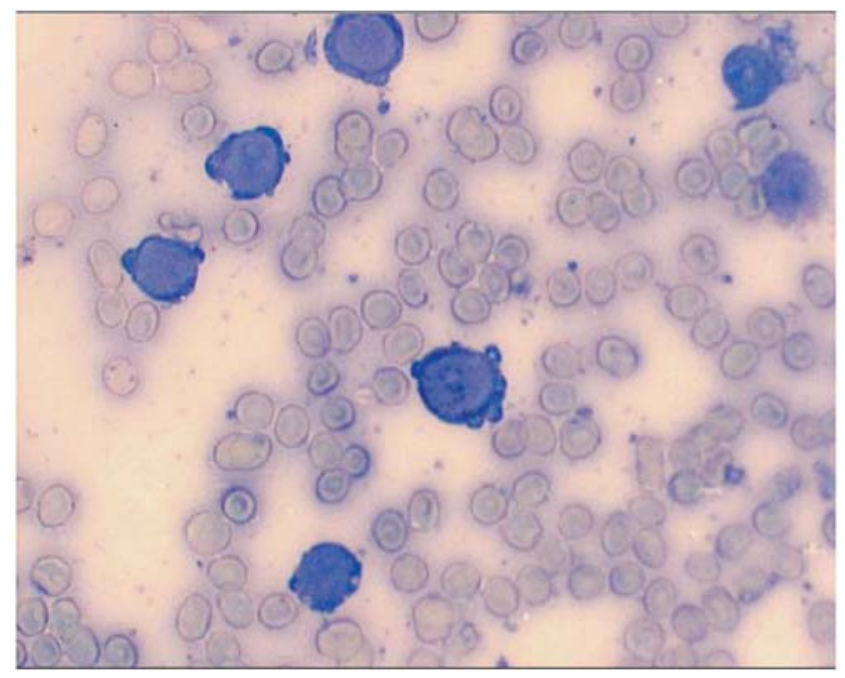

Figura 2. Distensão de medula óssea (x500). Blastos com coloração citoquímica Sudan Black positiva
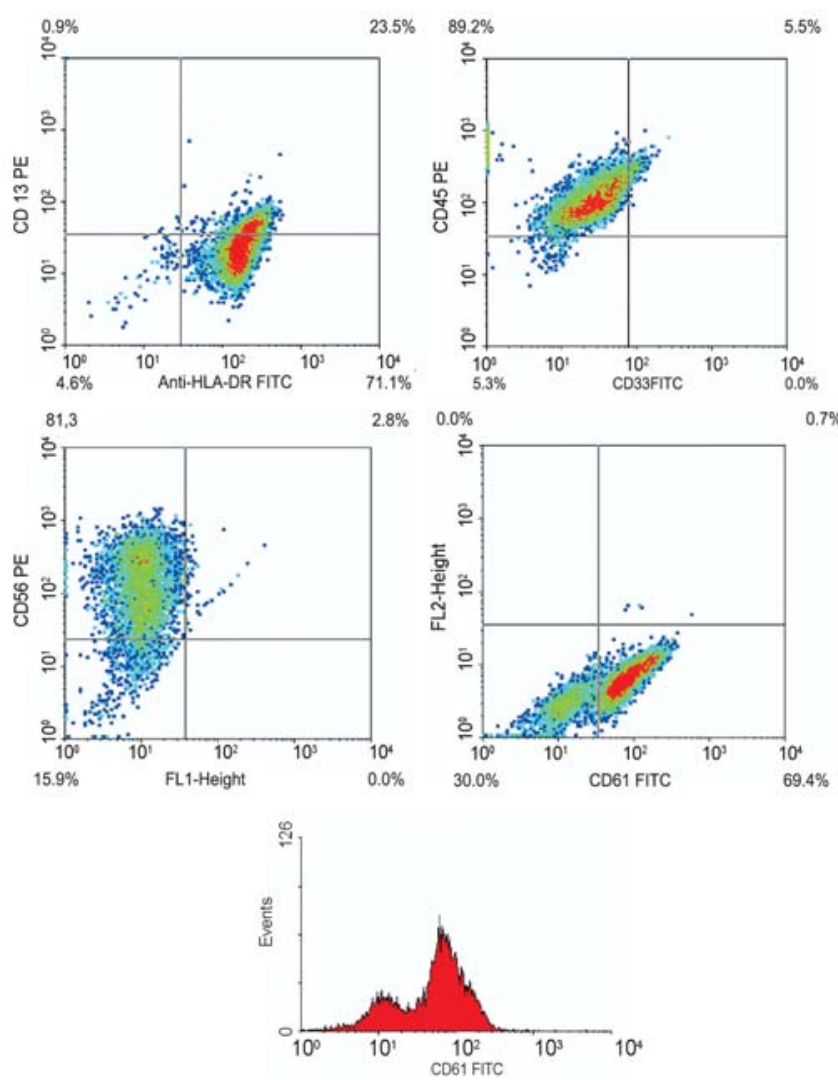

Fig. 3: Resultados da análise de dados adquiridos em citômetro de fluxo FACSCalibur (BD), usando-se o programa CellQuest (BD). Adquiridos 10.000 eventos. 


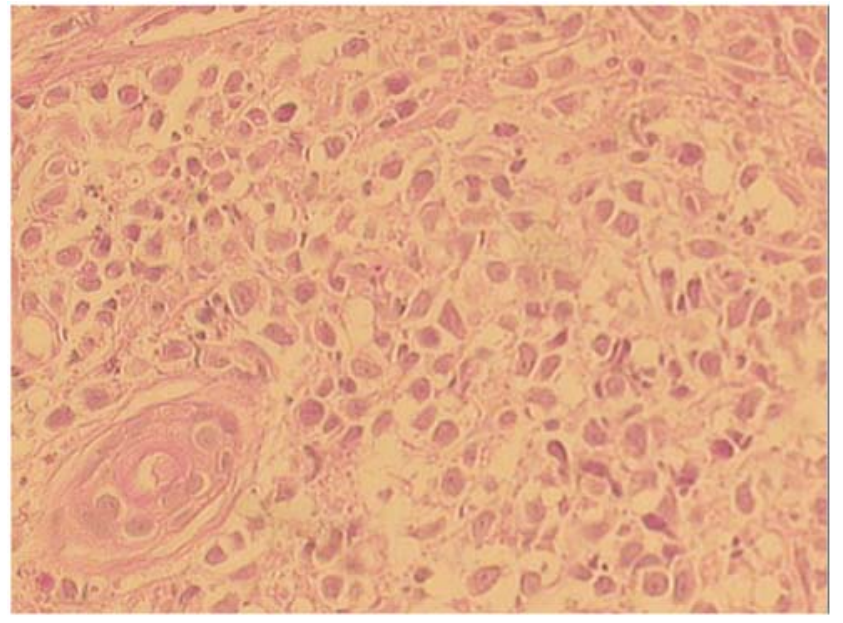

Fig 4 . Biópsia de pele (x400), revelou infiltrado mononuclear atípico consistente, com infiltração por leucemia

\section{Discussão}

A presença de $86 \%$ de blastos na medula óssea caracterizou uma leucemia aguda, segundo os critérios atuais da Organização Mundial da Saúde (OMS) e FAB. ${ }^{18-21}$ Para a LMA-M7, a OMS utiliza, como critério diagnóstico, a presença de $50 \%$, ou mais, de blastos dessa linhagem entre as células nucleadas da medula óssea. ${ }^{20}$

A imunofenotipagem, com a identificação de glicoproteínas de membrana CD41, CD42 e/ou CD61, é considerada o método de escolha para o diagnóstico de leucemia

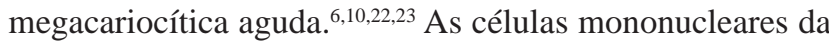
medula óssea, analisadas por Citometria de Fluxo, revelaram o seguinte imunofenótipo: 94\% CD45+ (marcador Panleucocitário); 93\% HLA-DR+ (marcador de células apresentadoras de antígeno-macrófagos, linfócitos $\mathrm{B}$ ativados e ainda mieloblastos); 81\% CD56+ (marcador células NK) e 69\% CD61+ (marcador de plaquetas e megacarioblastos). Adicionalmente foi identificada uma pequena subpopulação com fenótipo mielóide imaturo HLA-DR/CD13+ (23\%), mieloperoxidase positiva (11,5\%) e CD33+ (5\%). Os marcadores de linhagem linfóide T (CD3), de linhagem linfóide B (CD19 e CD10), de monócitos (CD14), marcador de células progenitoras hematopoéticas (CD117) e TdT foram negativos. Os marcadores CD41 e CD42 não foram testados.

A coloração positiva para Sudan Black (40\%) e a presença de marcadores como CD33 são compatíveis com a presença de envolvimento mielóide. Esses achados citológicos indicam que a LMA-M7 pode, freqüentemente, representar uma proliferação multilinhagem. ${ }^{7}$ No paciente analisado houve positividade para SB e presença de uma subpopulação adicional, o que sugeriu um comprometimento mielóide associado.

O caso relatado apresentou positividade para CD56, cuja expressão aberrante em algumas leucemias mielóides é indicativo de mau prognóstico, independente do carió- tipo. ${ }^{12,16,23,24} \mathrm{~A}$ expressão desse marcador pode estar associada à infiltração leucêmica extramedular, como lesões de pele, uma vez que se trata de uma molécula de adesão, a qual proporciona uma maior mobilidade para as células..$^{12,14} \mathrm{O}$ mecanismo de invasão extramedular das células leucêmicas é desconhecido; uma hipótese é que o CD56 expresso nos blastos pode se ligar à integrina beta 3 nas células endoteliais e ser responsável pela manifestação extramedular nas leucemias CD56+ e/ou que o crescimento de células CD56 são estimuladas por células NK na medula óssea, embora alguns autores não confirmem esta hipótese. ${ }^{17}$

Fenótipos aberrantes podem estar associados a diferentes subtipos de leucemias e deverão ser mais bem estudados para aumentar o entendimento biológico da doença. ${ }^{25}$

Com base em todos os resultados acima descritos, foi possível fazer o diagnóstico de LMA-M7 no paciente. Em virtude do mau prognóstico dessa patologia e da situação clínica desfavorável, o tratamento foi sintomático. Um mês após o diagnóstico, o paciente evoluiu para sepse, falência múltipla de órgãos e óbito.

\begin{abstract}
AML-M7 is a rare subtype of acute myeloid leukemia (AML). It is frequently associated with myelofibrosis and corresponds to a poor prognosis subtype. It rarely presents with infiltration at extramedullary sites. The bone marrow aspirate or biopsy identifies pleomorphic and basophilic cell populations that may present with cytoplasmatic projections. The use of immunophenotyping is essential for the diagnosis of AML-M7. The characteristic immunophenotype presents a leukemic cell population without most lymphoid and myeloid surface markers, but with an expression of the megakaryocytic antigens: CD41a (glycoprotein complex IIb/IIIa), CD42b (glycoprotein Ib) and/or CD61 (glycoprotein IIIa), or the factor VIII-related antigen. The cytochemical stain Sudan Black is frequently negative for megakaryocytic blasts; in this case, it was positive in $40 \%$ of the analyzed cells. The presence of CD56, whose aberrant expression in some myeloid leukemias indicates poor prognosis, might be associated with skin infiltration. Rev. bras. hematol. hemoter. 2007;29(2):185-188.
\end{abstract}

Key words: Acute megakaryocytic leukemia; cytochemistry; immunophenotyping; CD56; cutaneous infiltration.

\section{Referências Bibliográficas}

1. Martins SLR, Falcão RP. A importância da imunofenotipagem na leucemia mielóide aguda. Rev Ass Med Brasil. 2000;46(1):57-62.

2. Greer JP, Foerster J, Lukens J, et al. Wintrobe's Clinical Hematology, Chapter 79, 11th ed, Philadelphia 2004.

3. Garderet L, Labopin M, Gorin NC, et al. Hematopoietic stem cell transplantation for de novo acute megakaryocytic leukemia in first complete remission: a retrospective study of the European Group for blood and marrow Transplantation (EBMT). Blood. 2005;1005:405-9.

4. Moriarty AT. Acute and chronic leukemias. In: Rodak BF. Diagnostic Hematology. 1997;23(1):343-4. 
5. Atale UH, Razzouk BI, Raimondi SC et al. Biology and outcome of childhood acute megakaryoblastic leukemia: a single institution's experience. Blood. 2001;97(12):3727-32.

6. Pagano L, Pulsoni A, Vignetti M et al. Acute megakaryoblastic leukemia: experience of GIMEMA trials. Leukemia. 2002;16: 1622-6.

7. Cuneo A, Cavazzini F, Castoldi G. Acute megakaryoblastic leukemia (AmegL), M7 acute non lymphocytic leukemia (M7-ANLL). Atlas Genet Cytogenet Oncol Haematol. November 2003. URL: http:/ /www.infobiogen.fr/services/hromcancer/Anomalies/M7ANLLID 1100.html.

8. Bennet JM, Catovsky D, Daniel M, et al. Criteria for the diagnosis of acute leukemia of megakaryocytic lineage (M7). A report of the French-American-British cooperative assays. Ann Int Med. 1985;103:460-2.

9. Casasnovas RO, Slimane FK, Garand R, et al. Immunological classification of acute myeloblastic leukemias: relevance to patient outcome. Leukemia. 2003;17:515-27.

10. Kaleem Z, Crawford E, Pathan MH, et al. Flow cytometric analysis of acute leukemias - Diagnostic utility and critical analysis of data. Arch Pathol Lab Med. 2003;127(1):42-8.

11. Nishimura Y, Miyazawa T, Ikeda Y, et al. Molecular cloning and sequencing of feline CD56 (N-CAM). Eur J Immunogenet. 1999; 26:29-32.

12. Loughran Jr TP. CD56 hematologic malignancies. Leukemia Res 1999;23:675-6.

13. Bona ED, Sartori R, Zambello R, et al. Prognostic significance of CD56 antigen expression in acute myeloid leukemia. Haematologica 2002;(8):250-6.

14. Chang H, Brandwein J, Yi QL, et al. Extramedullary infiltrates of AML are associated with CD56 expression, 11q23 abnormalities and inferior clinical outcome. Leukemia Res. 2004;28:1007-11.

15. Agis H, Weltermann A, Fonatsch C, et al. A comparative study on dermographic, hematological, and cytogenetic findings and prognosis in acute myeloid leukemia with and without leukemia cutis. Ann Hematol. 2002;81:90-5.

16. Bekkenk MW, Jansen PM, Meijer CJLM, et al. CD56+ hematological neoplasms presenting in the skin: a retrospective analysis of 23 new cases and 130 cases from the literature. Ann Oncol. 2004;15:1097-108.

17. Graf M, Reif S, Hecht K, et al. High expression of costimulatory molecules correlates with low relapse-free survival probability in acute myeloid leukemia (AML). Ann Hematol. 2005;84:287-97.

18. Harris NL, Jaffe ES, Diebold J, et al. World Health Organization classification of neoplastic diseases of the hematopoietic and lymphoid tissues: Report of the Clinical Advisory Committee meeting - Airlie House, Virginia, November 1997. J Clin Oncol. 1999;17(12):3835-49.

19. Mckenna RW. Multifaceted approach to the diagnosis and classification of acute leukemias. Clin Chem. 2000;46,8 (B): 1252-9.

20. Rego EM, Falcão RP. Leucemia mielóide aguda - Diagnóstico: morfologia, imunofenótipo e citogenética. Ser Monogr Esc Bras Hematol. 2002;9:54-65.

21. Vardiman JW, Harris NL, Brunning RD. The World Health Organization (WHO) Classification of the myeloid neoplasms. Blood. 2002;100, 7:2292-302.

22. Talman MS, Neuberg D, Bennett JM, et al. Acute megakaryocytic leukemia: the Eastern Cooperative Oncology Group Experience. Blood. 2000;26(7):2405-11.

23. Basso G, Buldini B, De Zen L, et al. New methodological approaches for immunophenotyping acute leukemias. Haematologica 2001; 87(7):675-92.
24. Raspadori D, Damiani D, Michieli M et al. CD56 and PGP expression in acute myeloid leukemia: impact on clinical outcome. Haematologica. 2002;(87):1135-40.

25. Bahia DMM, Yamamoto M, Chauffaille MLLF et al. Aberrant phenotypes in acute myeloid leukemia: a high frequency and clinical significance. Haematologica. 2001;86(8):801-6.

Avaliação: Editor e dois revisores externos Conflito de interesse: não declarado

Recebido: 28/12/2006

Aceito após modificações:05/01/2007 